\title{
Analysis of Super-cooled Water Droplet Impact on a Thin Water Layer and Ice Growth.
}

\author{
M. Quero ${ }^{(1)}$, D. W. Hammond ${ }^{(1)}$, R. Purvis ${ }^{(2)}$ and F.T. Smith $^{(3)}$ \\ ${ }^{(1)}$ Cranfield University, Bedfordshire, UK, MK43 OAL \\ ${ }^{(2)}$ University of East Anglia, Norwich, UK, NR4 7TJ \\ ${ }^{(3)}$ University College, London, UK, WC1E 6BT
}

\begin{abstract}
The mechanics and heat flow of the impact and freezing of a Super-cooled Large Droplet (SLD) on to a thin mobile water layer is considered in the parameter range of interest to airframe icing. A series of 2-D Navier-Stokes solutions for droplet impact are presented. These simulations show the formation, ejection and break-up of splashed off jets and the freezing process. The simulations are compared critically to experiments performed under similar conditions.
\end{abstract}

\section{Notation section}

$\begin{array}{ll}\mathrm{Re} & \text { Reynolds number } \\ \mathrm{We} & \text { Weber number } \\ \mathrm{H} & \text { Water film thickness } \\ \mathrm{R} & \text { Droplet diameter } \\ \varepsilon & \text { H/R } \\ \rho & \text { Fluid density } \\ \vec{u} & \text { Velocity vector } \\ p & \text { Pressure field } \\ v & \text { Kinematic viscosity } \\ T & \text { Temperature } \\ T_{\mathrm{m}} & \text { Melting temperature } \\ C_{p} & \text { Specific heat } \\ k_{c} & \text { Thermal conductivity } \\ L & \text { Latent heat } \\ k_{\mathrm{T}} & \text { Thermal conductivity } \\ \mathbf{m}_{\mathrm{i}, \mathrm{j}} & \text { Normal vector to ice-liquid interface } \\ \mathrm{W} & \text { Droplet width } \\ \mathrm{h} & \text { Droplet height } \\ \mathrm{VED} & \text { Volume Equivalent Diameter }=\sqrt[3]{w^{2} \cdot h} .\end{array}$

\section{Introduction}

The design of aircraft today relies extensively on numerical tools to assess the influence of icing on the performance of aerodynamic surfaces and propulsion systems. As part of the development of these tools, there is much interest in what happens as the droplet first makes contact with the aircraft (or other structure). This focus has increased with the acceptance that droplet size may be a significant factor beyond the consideration of the trajectory of droplets around the aircraft.

Thoroddsen (2002) reports on an experimental study of single droplet impacts onto undisturbed water films. His experiments cover droplet Re 
numbers of up to 4000. This is an order of magnitude lower than the situation under investigation here but he has given the field a valuable range of data including jet velocities as a function of droplet size and speed for normal impact and the influence of viscosity. In particular Thoroddsen provides evidence of the formation of a fast moving horizontal jet in the early stages of the impact which was to prove supportive to work on modelling droplet impact.

Weiss and Yarin (1999) provide a numerical study of a single water droplet impact onto a film of the same liquid. They consider surface tension and gravity but not viscosity or compressibility. The results provide information of the velocity of the jets emanating from the contact area of the droplet with the film at low Weber numbers and at low droplet velocities. Their results agree well with the experimental work by Thoroddsen suggesting that in this range of Weber number and speed and in the case the droplet properties are the same as layer properties, viscous and compressibility effects may be neglected. Further background is provided by Howison et al. (2004) with an analytical study of the high speed droplet impact on a shallow water layer giving special emphasis to the solution at very short timescales. They only consider the case when $\varepsilon=H / R$ is small and $u$ is very large where $H$ is water film thickness and $\mathrm{R}$ the droplet diameter.

Josserand el al. (2003) purses a similar line but looks at intermediate time scales giving results on the form of the jets as they begin to project upwards away from the surface of the water layer/target. Their results show the influence of the Re number on the form of the jet.

The current investigation follows on from the work of Purvis et al. (2004). He studies droplet impact onto an undisturbed water layer address the parameter range associated with SLD icing. Surface tension, viscosity, gravity and the influence of pre-existing air flow are neglected. The work provides and discusses solutions for the flow over intermediate timescales for different angles of impact varying droplet sizes, velocities and layer depth. He also starts to consider methods of quantifying the amount of fluid displaced from the surface. Purvis' work has been expanded upon in our study by a number of refinements including the ability to ascribe different properties to the droplet and the layer. In particular, some of the physical properties are made to be dependant on temperature and a thermal model is included. In this way variations in local fluid properties may be accounted for. The model also provides for the freezing and the development of ice roughness elements on the target surface.

The authors have previously reported experimental observations of SLD impact Hammond et al. (2005) and these are used to compare and contrast with the results from the new model.

\section{The Physical Problem}

The experiments provide images of the impacts and subsequent splashes. A wide range of variables has been used in order to give a general idea of how the corona develops for a range of the droplet sizes, droplet temperatures, velocities of impact, angles of impact and layer depths (see table 1).

\begin{tabular}{|c|c|}
\hline Variable & $\begin{array}{c}\text { Range of } \\
\text { interest }\end{array}$ \\
\hline Droplet diameter & 40 to $400 \mu \mathrm{m}$ \\
\hline Velocity & 20 to $120 \mathrm{~m} / \mathrm{s}$ \\
\hline Water film thickness & 10 to $100 \mu \mathrm{m}$ \\
\hline Water film velocity & $1-10 \mathrm{~m} / \mathrm{s}$ \\
\hline Angle of impact & $10^{\circ}$ to $90^{\circ}$ \\
\hline Droplet Temperature & $+20^{\circ}$ to $-20^{\circ} \mathrm{C}$ \\
\hline Film Temperature & $+20^{\circ}$ to $0^{\circ} \mathrm{C}$ \\
\hline
\end{tabular}

The pictures shown in Figures $1 \& 2$ (Hammond et al.2005) give examples of sequences of impact images chosen to show the initial droplet, the corona at its most fully developed and the subsequent break-up of the corona. The time taken for the corona to reach its greatest size in these two cases is approximately 500 and $400 \mu \mathrm{s}$ respectively.

The main difference between the impacts depicted in figures $1 \& 2$ is the speed of the droplet and or the air flow. In figure 1, the incoming droplet is relatively spherical and the walls of the resulting corona are more smooth and conical than in the higher speed case. Also the satellite droplets generated as the corona breaks down are noticeably larger. In the higher speed case, the distortion of the droplet prior to impact is more noticeable. The general shape of the corona has changed but its overall height and width at the time it starts to break up is similar. 


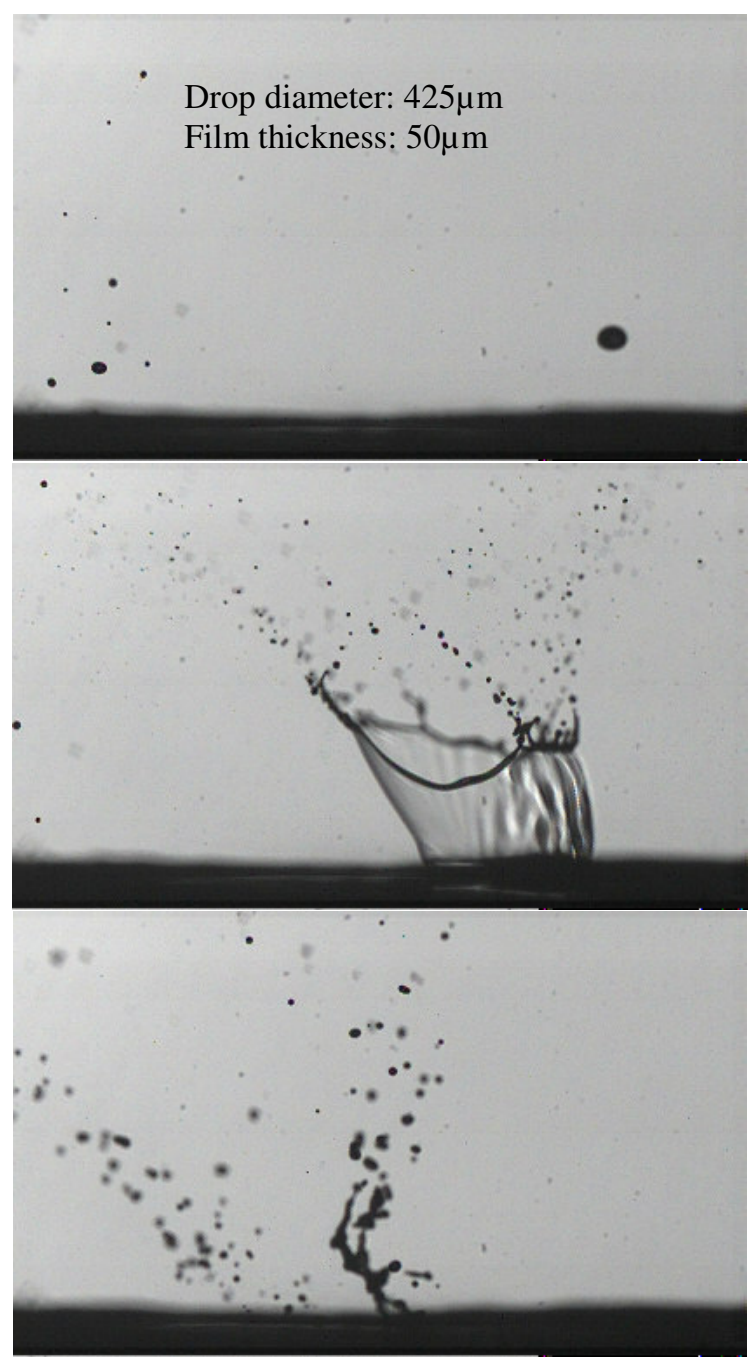

Figure 1. Impact at $24 \mathrm{~m} / \mathrm{s}, 70^{\circ}$ and ambient temperature. $50 \mu \mathrm{m}$ film.

These coronas are of the order of 2 to $3 \mathrm{~mm}$ in height and width.

\section{Description of the Model}

The model is based on VOF methods which calculate the solution of two dimensional transient fluid flow with free boundaries (Hirt et al. 1981, Torrey et al. 1985 and Nichols et al. 1981). These methods have developed over time and have used previously to simulate the droplet impacts. The main idea of the VOF method is to track the position of the free boundaries by defining a function $\mathrm{F}(x, y, t)$ over every cell of the computational mesh. The value of $F$ in a cell is equal to the fraction of volume of the cell occupied by the fluid. Therefore:
Drop diameter: $592 \times 220 \mu \mathrm{m}$

Film thickness: $50 \mu \mathrm{m}$

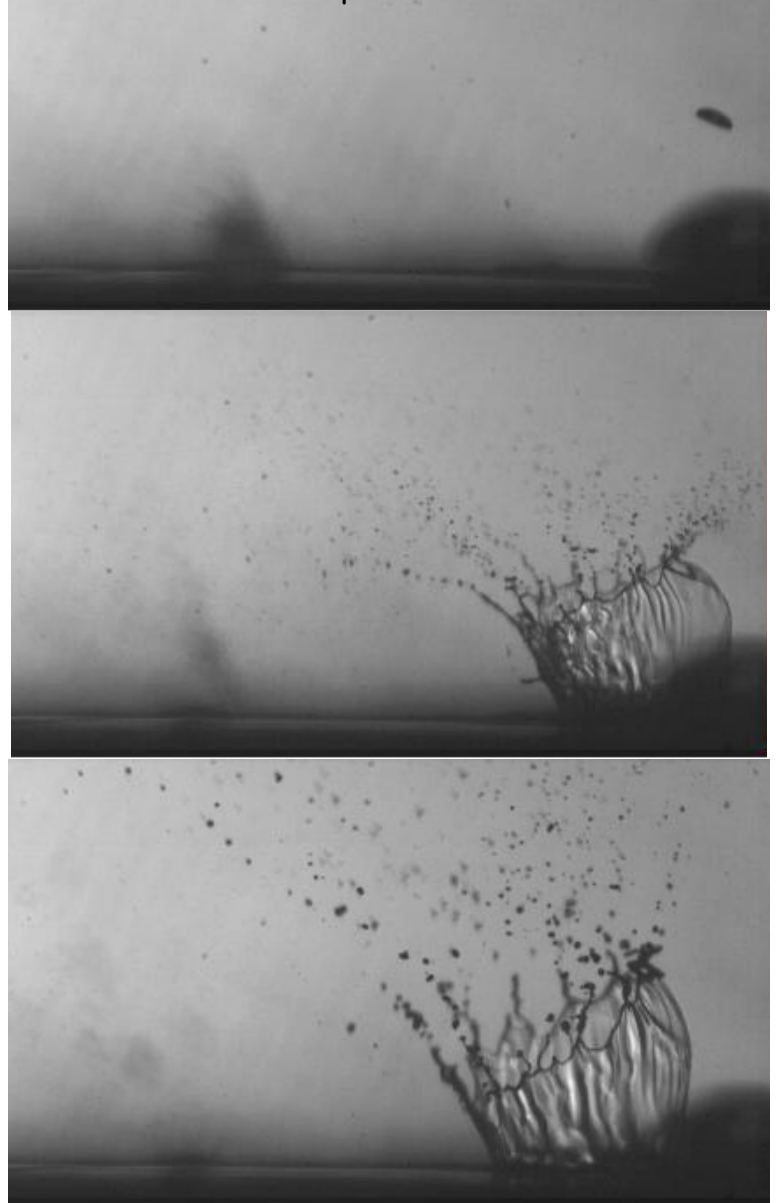

Figure 2. Impact at $49 \mathrm{~m} / \mathrm{s}, 70^{\circ}$ and $-9^{\circ} \mathrm{C} .50 \mu \mathrm{m}$ film.

- $\quad \mathrm{F}(x, y, t)=1$ if the cell is completely full of fluid.

- $\quad \mathrm{F}(x, y, t)=0$ if the cell has no fluid.

- $\quad \mathrm{F}(x, y, t) \in(0,1)$ if the cell contains a boundary.

A cell is considered to be a boundary cell if it has at least one neighbouring cell with $\mathrm{F}=0$.

The fluid equations to be solved are the NavierStokes equations: 


$$
\begin{aligned}
& \frac{\partial u}{\partial t}+u \frac{\partial u}{\partial x}+v \frac{\partial u}{\partial y}=-\frac{1}{\rho} \frac{\partial p}{\partial x}+g_{x}+v\left[\frac{\partial^{2} u}{\partial x^{2}}+\frac{\partial^{2} u}{\partial y^{2}}\right] \\
& \frac{\partial v}{\partial t}+u \frac{\partial v}{\partial x}+v \frac{\partial v}{\partial y}=-\frac{1}{\rho} \frac{\partial p}{\partial y}+g_{x}+v\left[\frac{\partial^{2} v}{\partial x^{2}}+\frac{\partial^{2} v}{\partial y^{2}}\right]
\end{aligned}
$$

Velocity components $(u, v)$ are in Cartesian coordinates $(x, y)$. The acceleration is denoted by $\left(g_{\mathrm{x}}, g_{\mathrm{y}}\right), p(x, y)$ is the pressure field, $v$ is the kinematic viscosity and $\rho$ is the fluid density.

The momentum equation is defined by:

$$
\frac{\partial u}{\partial x}+\frac{\partial v}{\partial y}=0
$$

It is relevant in (1) the fact that the viscosity is included in the calculations. Some other authors have neglected it but, because this work considers two different liquids due to the difference of the temperatures in both of them, viscosity is here taken into account and is made temperature dependent. In the same way, some other variables have been included in the code because the needed that came up when dealing with heat transfer and freezing. These variables are specific heat $C_{p}$, thermal conductivity $k_{c}$ and thermal diffusivity $k$. The surface tension has been included by previous authors although for this work it has been done temperature dependent too. The reason this has been done is that it has been found that some properties change substantially when dealing with supercooled temperatures for water, especially the viscosity (Hallett, 1963, Trinh et al, 1994).

In order to calculate the heat balance during the impact and splash, equation (3) has to be also solved. This provides the values of the temperature in every computational cell at every time step and thereby permits the determination of the local values of the physical properties which depend on it. The boundary conditions force that there is not going to be heat transfer between the fluids and the void, and the initial conditions give a first temperature to the droplet and to the layer.

$$
\begin{aligned}
& T_{t}+\vec{u} \cdot \nabla T=k\left(T_{x x}+T_{y y}\right) \\
& \frac{\partial T}{\partial \vec{n}}=0 \\
& T_{\text {Layer }}=\text { Initial layer }
\end{aligned}
$$

temperature.

$$
T_{\text {Droplet }}=\text { Initial droplet }
$$

temperature.

$\rightarrow$

$u$ is the velocity vector and $k$ is the thermal diffusivity.

\section{Freezing}

In order to simulate the freezing of part of the water, two new features are introduced to the model. The first provides a moving solid boundary (of ice) which perturbs the flow of the liquid. The second is that part of the water is able to change state with the attendant latent heat argument. A thin seed layer ice roughness is initially set at the bottom of the water layer. Heat is transferred across the ice-water interface causing the interface to move.

In order to model this, the heat transfer in those areas where the ice is in contact with the liquid fluid is calculated according to solidification theory (Davis, 2001). Figure 3 shows the temperature profile between the ice, layer and super-cooled droplet. The horizontal axis $x$ represents the distance and the vertical $T$ the temperature. At the first time-step when the droplet touches the layer, there is ice at temperature $T_{\mathrm{m}}$ (melting temperature) until the distance $h_{0}$. From this point starts the water layer. As the distance increases, the temperature increases until $h_{1}$ which is the point where the droplet contacts the film. Therefore there is a discontinuity on the temperature function representing the initial difference on the temperature values of both fluids. The heat transfer at distance $h_{1}$ is calculated resolving (3), however, at $h_{0}$ is equation (4) which calculates the temperature in the interface between the ice and the liquid. 


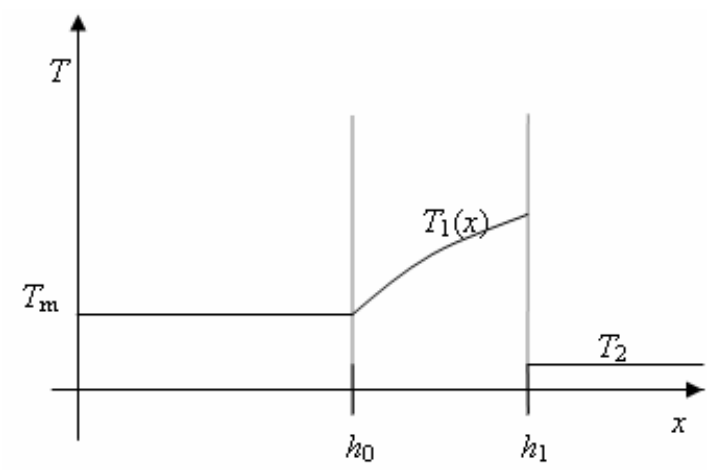

Figure3. Temperature profile for the case icelayer-droplet.

When the super-cooled droplet is in direct contact with the ice, i.e., $h_{0}=h_{1}$, the temperature is calculated according to (4) too.

Solving (4) the moving boundary $h(t)$ formed by the ice is also determined at every time-step. This term allows obtaining the amount of increasing of ice when the temperature in the cell drops bellow $T_{\mathrm{m}}$.

$$
\begin{aligned}
T^{S}=T_{m} & \text { In solid, } x<h_{0} \\
T_{t}^{l}=k^{l} T_{x x}^{l} & \text { In liquid, } x>h_{0} \\
T^{l}=T^{S}=T_{m} & \text { On } x=h_{0} \\
\rho^{S} L \dot{h}=-k_{T}^{l} T_{x}^{l} &
\end{aligned}
$$

Where $L$ is the latent heat and $k_{\mathrm{T}}$ is the thermal conductivity. Superscript $S$ refers to the solid and $l$ to the liquid.

Once the rate for the ice growth is calculated, the boundary is moved in the direction of its normal vector $\mathbf{m}_{\mathrm{i}, \mathrm{j}}$ which is determined according to the PLIC method (Scardovelli, 1999) as figures 4 'a' and ' $b$ ' illustrate.

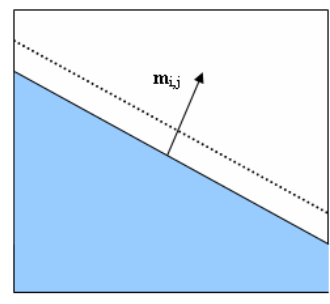

Figure 4a

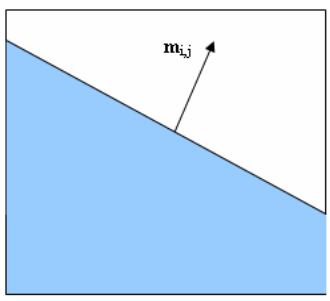

Figure $4 b$

\section{Results.}

Firstly, the model has been used to simulate the form of an impact event for which corresponding experimental data is available. It is important to remember that the experimental realm is 3-D whereas the simulation is 2-D. Whilst such comparisons are inherently limited, they provide some basis for the application of 2-D simulations.

Figure 5 a \& b shows one such comparison. Note that in the figure, the droplet shown in the simulation is travelling from left to right whereas in the image of the actual droplet, the droplet is travelling from right to left.

In the simulation, the initial droplet aspect ratio is set to correspond with the experimental data.

In the early stages of the splash, both the simulation and the experiment show a shallow cone of ejected material.

As the process unfolds, the corona becomes wider and more steep sided. It is possibly apparent, both in the simulation and the experimental image that the side of the corona or jet into which the droplet would move (had it not collided with the surface) contains more water.

As the splash evolves further the experimental corona shape begins to differ significantly from the form of the simulated jets. This is thought to be in part due to the influence of the air movement.

In the simulation, colour has been used to show the boundary between the droplet water and the layer water. It can be seen, as postulated by other workers, that the ejected water originates largely from the layer.

To continue the analysis further, the experimental images have been used to make a series of measurements of the scale and speed of the corona development and break-up. These are then compared directly with simulations made using appropriately matched input parameters.

The scale and angular measurements used are defined in figures 6 and 7. The velocities of the corona were determined by measuring the extension of the left and right sides of the corona between subsequent images, divided by the interframe time, as the corona begins to grow. 

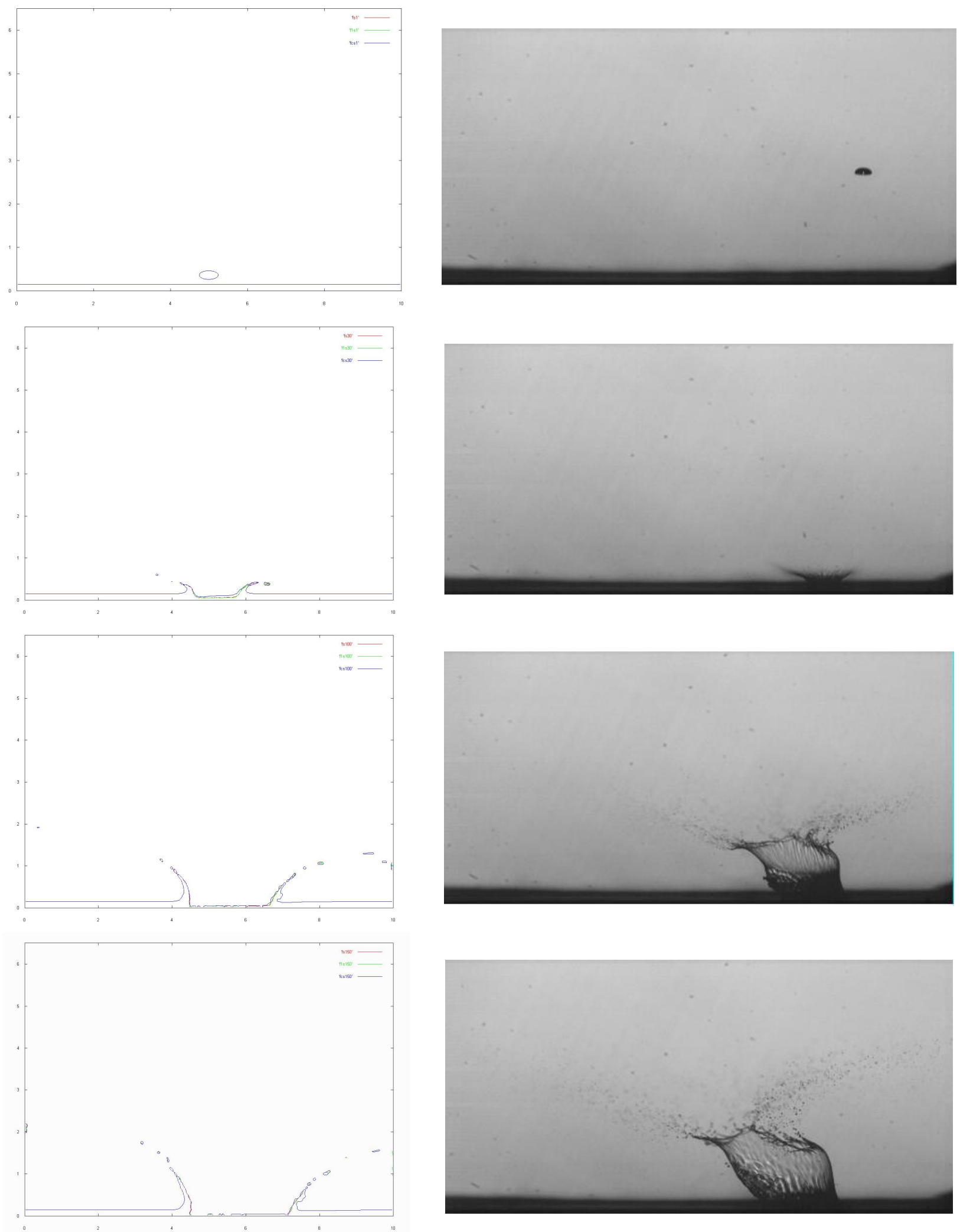

Figures $5 \mathrm{a}$ (free surface simulation) \& b (experiment). $70^{\circ}$ impact of a $483 \times 170 \mu \mathrm{m}$ droplet at $55 \mathrm{~m} / \mathrm{s}$ over a $50 \mu \mathrm{m}$ water film thickness and at ambient temperature. 


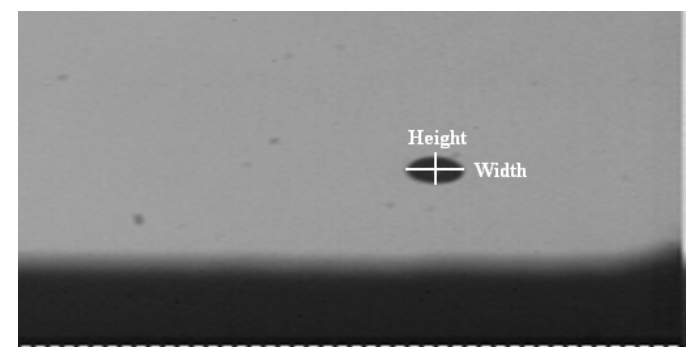

Figure 6. Width and height droplet measurement.
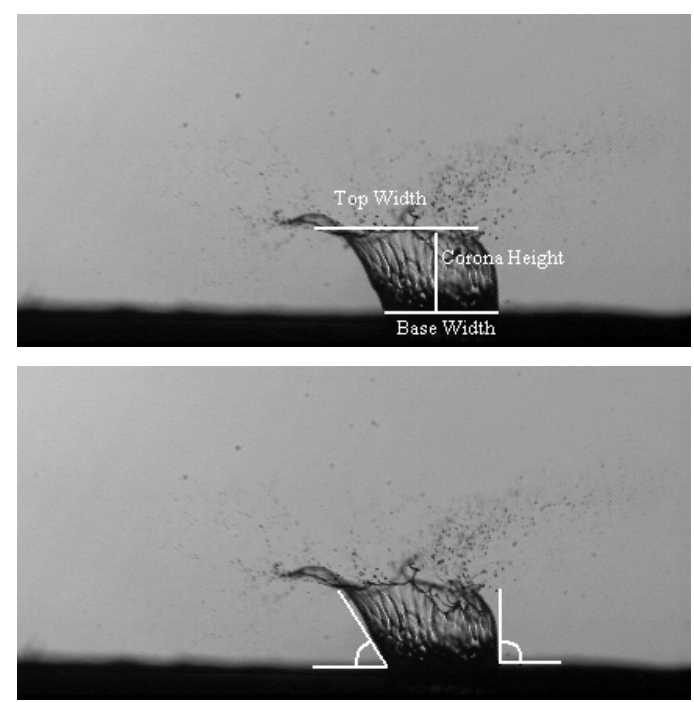

Figure 7a. Height, Top Width and Base Width. Figure $7 \mathrm{~b}$. Left and right angles.

A series of comparisons are now presented which show the level of agreement between the simulation and the experimental data in these terms.

\section{Velocity of the jets/corona}

The comparison is presented in figure 8. Is shows the simulated and observed values for the velocity of the two sides of the corona/jet. There is a significant level of scatter in the simulated values and the experimental ones. In the case of the experiment, it is noted that no two impact events are exactly the same. Additionally there is also a certain variation in the timing of the velocity measurement due to the random timing of the initial impact relative to the timing of the pair of images used to determine the velocity. In the case of the simulated data, the source of the scatter is due to the use of slightly different input variables which were initially felt to be of secondary influence (temporal and spatial resolution, \& droplet velocity). In spite of this it can be that the overall values are quite comparable. The experimental data indicates a trend positive trend of jet/corona velocity with increasing droplet size. This trend is so not clear in the simulated data because of the limited number of cases performed. A similar story emerges with the comparison of jet speed with droplet speed but the experimental trend is significantly weaker (Hammond 05).

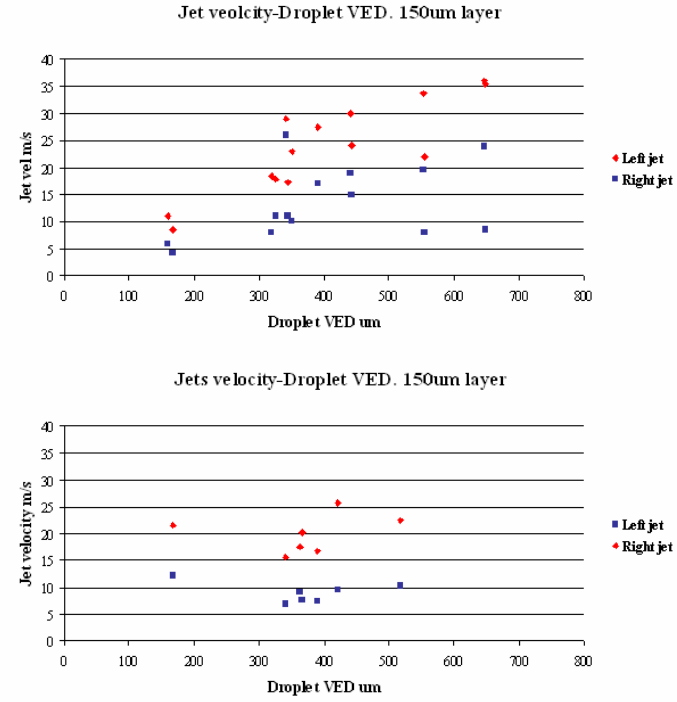

Figure 8. Jets velocity comparison. Up experiments and down modelling

\section{Jet/corona angle}

Figure 9 shows jet/corona side angles for the case of a 70 degree angle of impact. The experimental results show a significant level of scatter in the data but also indicate that the jet/corona angles are relatively insensitive to droplet size. The small trend that is apparent is that as the droplet diameters increase, the corona becomes more flattened. It is worth pointing out at this stage that the larger droplet impacts give rise to larger coronas and that this may mean that we observe a greater degree of aerodynamic distortion as droplet size increases. This could in part be responsible for the change in angles of both sides of the corona as droplet diameter increases. The values of the simulated angles agree quantitatively very well with the experiments but the trend of jet/corona angle with droplet size is not apparent. 
Jet angle-D roplet VED. 150um layer

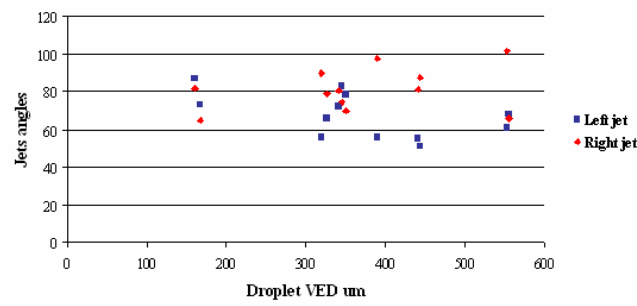

Jets angle-Droplet VED. 150um layer

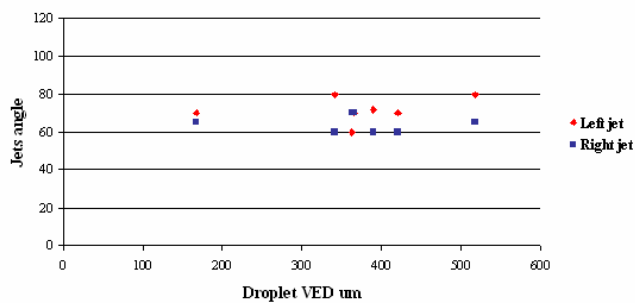

Figure 9. Jets angle comparison. Up experiments and down modelling

\section{Jet/corona size}

The last comparisons presented on the form of the jet/corona relate to the size the corona grows to before breaking down. In the experiment, the break-up of the corona occurs a s a result of a combination of surface tension and aerodynamic disturbance. The simulations, the aerodynamic forces are absent. In figure 10, it can be seen that for the experimental results the three measures of corona size all increase with droplet diameter and that they also maintain broadly similar proportions.

Corona size-Droplet VED. 150um layer

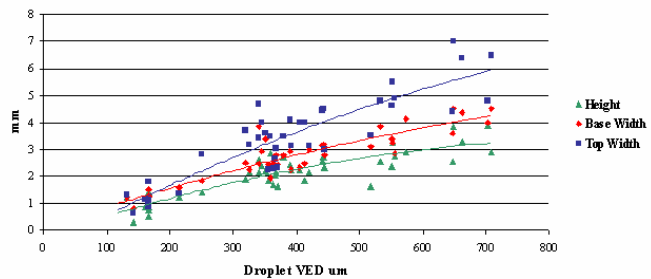

Corona Size- Droplet VED. 150um laye

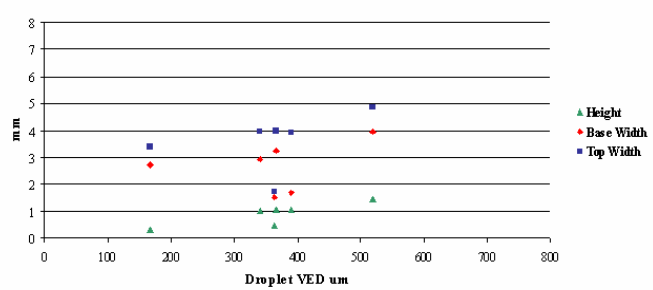

Figure 10. Corona size comparison. Up experiments and down modelling
For the simulations, again the level of quantitative agreement is good but that the ratio of height to the two width measurement appears to be significantly lower. It is apparent that the jet break-up process appears to begin earlier in the simulations than in the experimental observations. The definition used to define the point where the simulated jet begins to break up has been the time when the broken off droplets are of a size similar double the jet thickness. In effect this can mean that the onset of break-up in the simulations occurs even earlier than is shown in the figure 10. This shows that the modelling of the jet break-up is unrepresentative of the experimental data in this respect. One possible factor involved with this is that the simulation does not provide for the effect of the air flow in slowing down the spread of the jet being projected into the airflow. It is this jet which breaks up in the simulations first. It is also possible that this may be a difficulty with applying a 2-D simulation to a 3-D phenomenon or it could be that the quasi static surface tension term used is not appropriate for this dynamic situation.

\section{Temperature.}

One important aspect of the ejection of water from an aerofoil surface, which may be heated, is the temperature of the ejected liquid as a function of the temperature of the incoming droplet and water layer present on the surface. The model has been used to quantify this. Also since the fluid properties are temperature sensitive and model as such, the performance of the thermal analysis is important. Some experimental data exits for the impact of supercooled water droplets (Hammond 05)

The following case is typical of any of the simulations reported here but presented complete with the calculated local temperatures.

Figure 11 shows the thermal history of a simulation of an impact of a super-cooled water droplet at $-10^{\circ} \mathrm{C}$ and a water film at $15^{\circ} \mathrm{C}$. The droplet VED is $240 \mu \mathrm{m}$ and the water layer thickness is $50 \mu \mathrm{m}$.

The figure shows that, in the early stages of the splash, the temperature in the impact area essentially the temperature of the incoming droplet. The right jet, which contains a significant amount of water from the droplet, is 

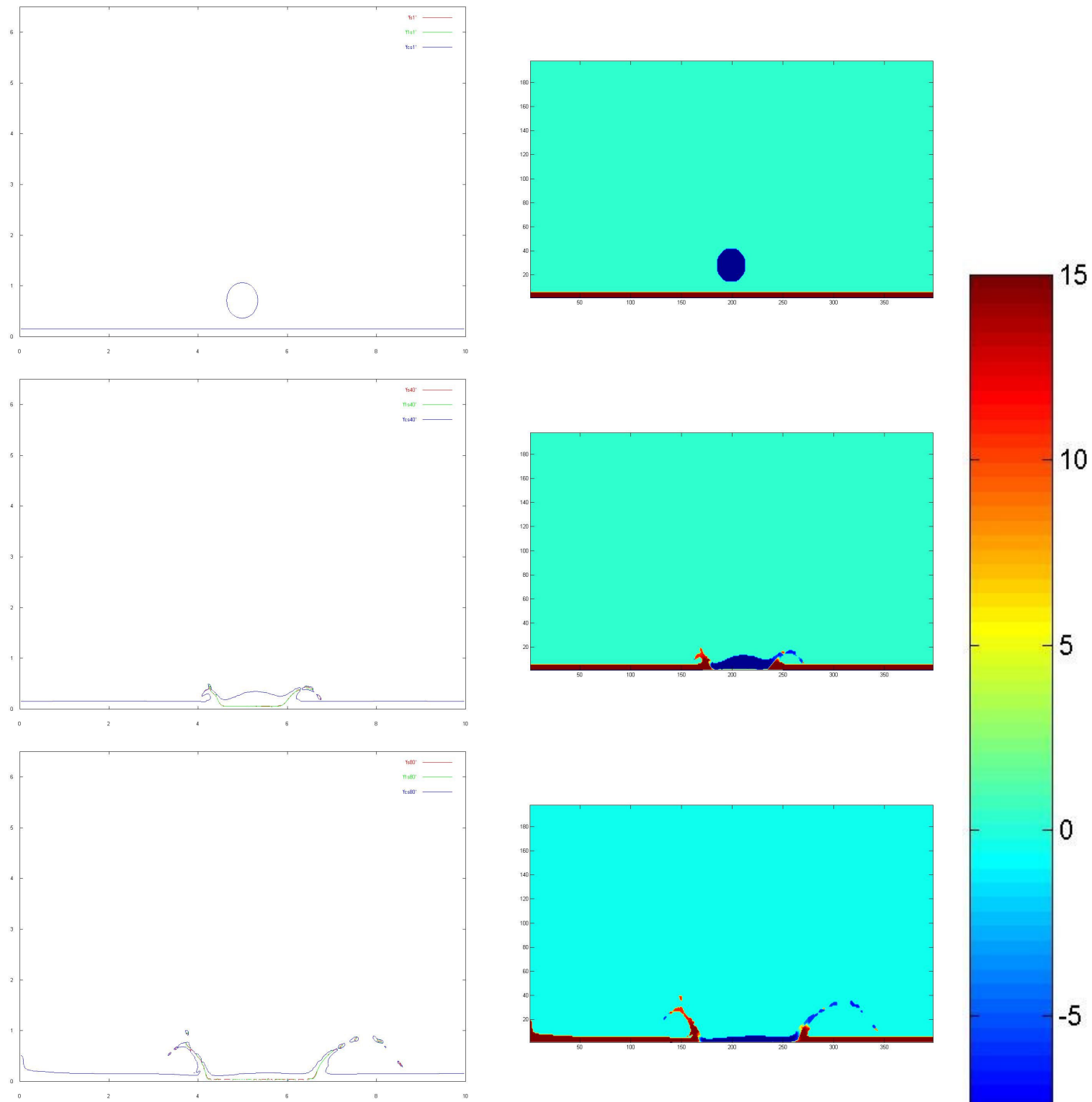

0

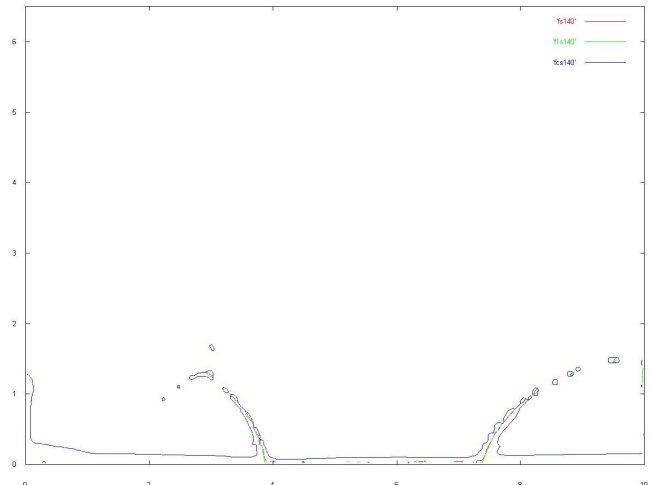

Figure 11a. Free surface

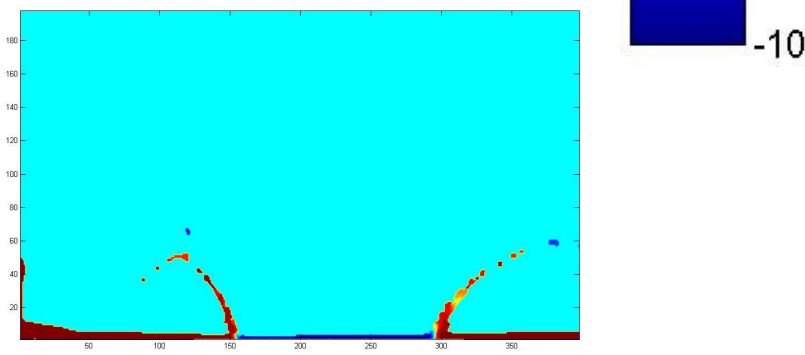

Figure 11b. Temperature distribution 
significantly cooler than the left jet. Indeed the right jet it is super-cooled.

As the splash evolves it is observed that the spreading impact zone remains super-cooled temperatures and the right jet is continues to be cooler than the left jet. As time proceeds, and with the inclusion of more layer water into both jets, the right jet warms up and assumes a similar temperature to the left jet. The water in the impact area remains super-cooled.

\section{Solidification results.}

The first illustration of the use of this model for freezing presents a simulation of an array of ice crystals submerged in a water film. In figure 12 the array of triangular seed crystals are seen to grow into the otherwise undisturbed water film. It can be seen isolated crystals grow into each other and then as the ice front grows into the fluid it becomes smoother. In this case the ice crystal temperature is set to $-0.1^{\circ} \mathrm{C}$ and the layer is set at $+1^{\circ} \mathrm{C}$.

With a freezing model developed, it is now possible to couple the solidification with the thermal droplet impact method to simulate more of the icing process.

This is illustrated in figure 13 which shows a simulation of the normal impact of an oblate super-cooled water droplet at $-10^{\circ} \mathrm{C}$ on a thin water film at $1^{\circ} \mathrm{C}$. There are 50 ice crystals set at a temperature of $-0.1^{\circ} \mathrm{C}$.

As the droplet approaches the film, the temperature of the layer in the vicinity of the impact site decreases and the ice growth rate increases. The triangular ice layer acts to modify the flow of the droplet and layer water and so affects the form of the splash.

As the simulation proceeds, the water layer in the vicinity of the impact cools further and the ice layer begins to grow into the super-cooled droplet water. The jet formation is a relatively slow process in relation to the freezing but the jets may be seen in the last frame presented of this simulation.

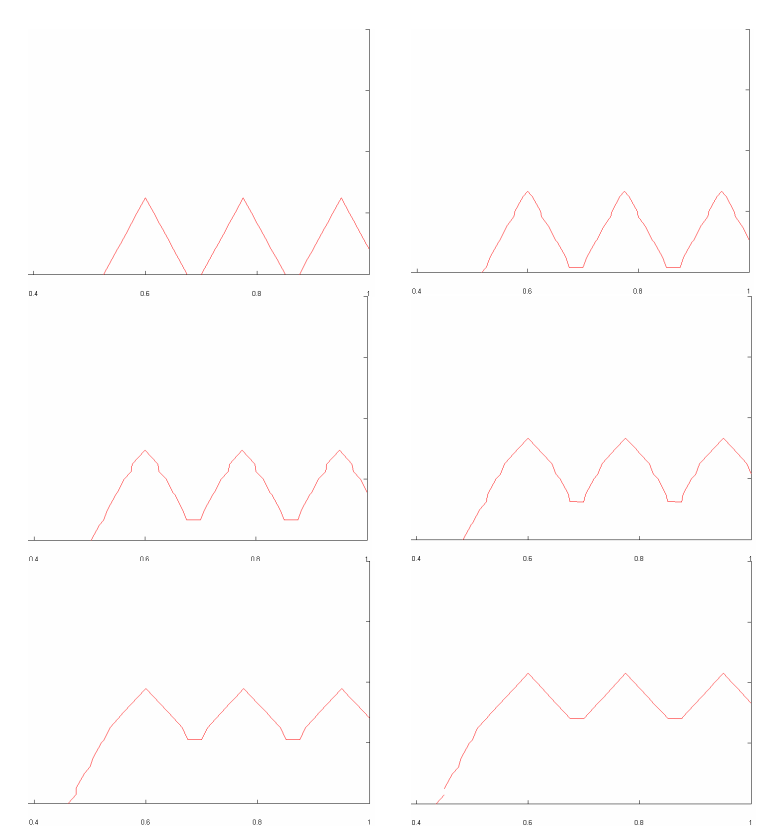

Figure 12. Detail of the ice crystals growth. 

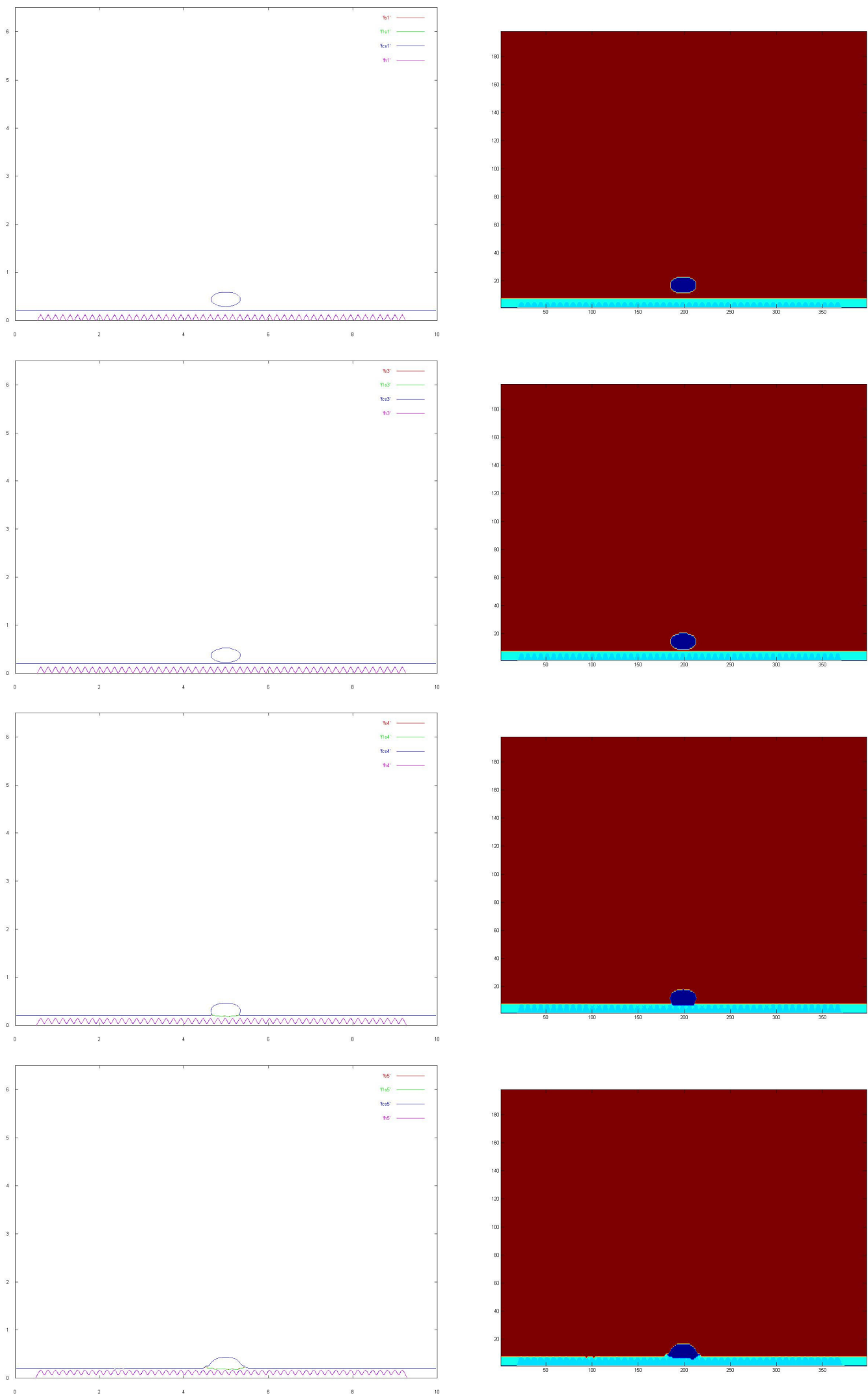

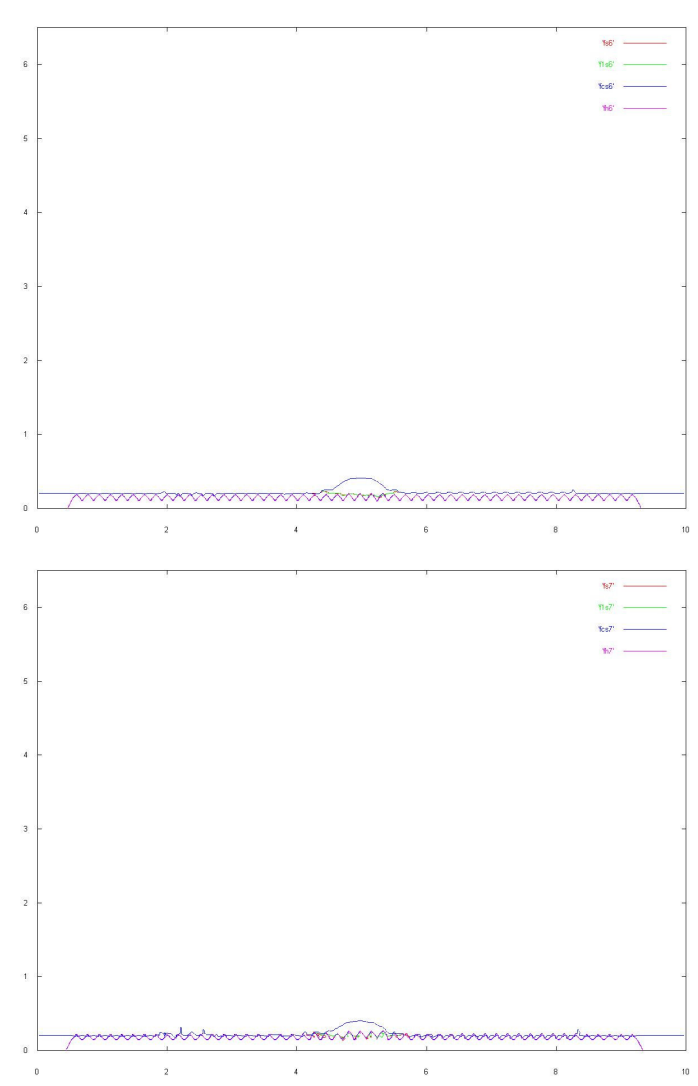

Figure 13a. Free surface

\section{Conclusions}

The simulations using the model provide a picture of the splash and freezing of SLD over a range of variables which are appropriate to aircraft icing. This is in spite of the fact that the model is a 2-D method and the experiments involve 3-D impact.

This picture agrees well quantitatively with corresponding experiments in respect of the velocity and initial trajectory of ejected water.

The break-up of the ejected jets and their trajectory at some time after the impact, as simulated, appears to differ from that observed experimentally. It would see that the influence of the air flow becomes apparent after the initial stages of the splash process.

Scatter in the experimental results can be on a similar magnitude to the difference between the simulated and the real data
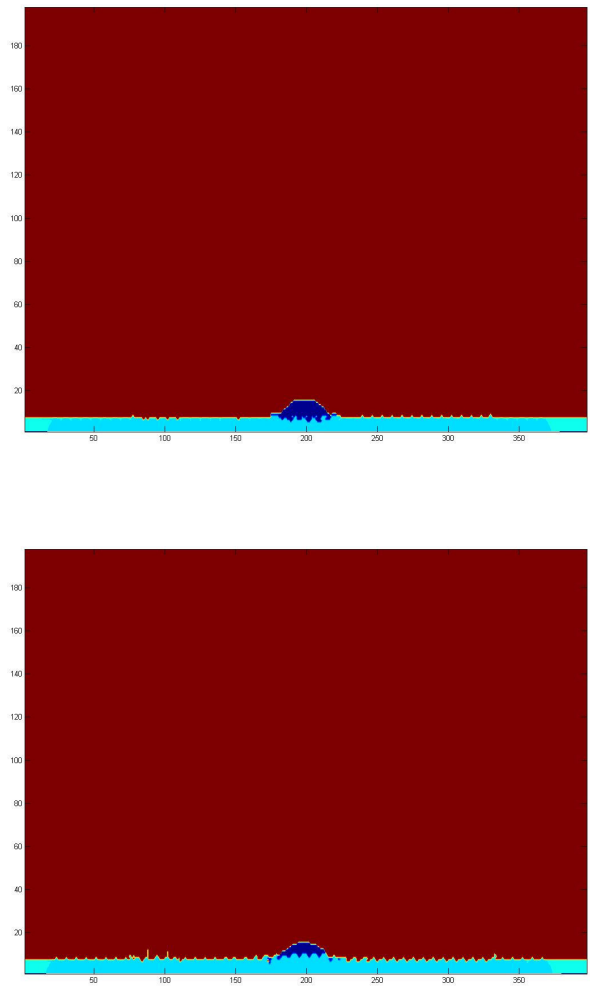

Figure 13b. Temperature profile

The model is providing information which cannot be compared with the experimental data on account of the technical problems associated with measuring temperatures in regions of great temporal and spatial thermal gradients.

\section{Acknowledgements.}

The authors would like to thank GKN Aerospace Services for the support through the Faraday Partnership for Industrial Mathematics, managed by the Smith Institute, to Dean Miller and Peter Tate from NASA Glenn and to Jason Tan from Wichita State University for their active collaboration during the experiments development in the Cranfield University High Speed Vertical Tunnel.

\section{References.}

S.H. Davis. Theory of Solidification. Cambridge University Press. 2001.

J. Hallett. The Temperature Dependence of the Viscosity of Super-cooled Water. Proceedings of Physical Society (1963), vol. 82, pp 1046-1050. 
D.Hammond, M.Quero, D.Miller, R.Purvis, O.McGregor and J.Tan. Analysis and Experimental Aspects of the Impact of Supercooled Water Droplets into Thin Water Layers. AIAA-2005-0077.

C.W. Hirt and B.D. Nichols. Volume of Fluid (VOF) Method for the Dynamics of Free Boundaries. Journal of Computational Physics (1981), vol. 39, pp. 201-225.

C. Josserand and S. Zaleski. Drop Impact on a Thin Layer. The Physics of Fluids (2003), vol. 15, pp. 1650-1657.

B. D. Nichols, C. W. Hirt and R. S. Hotchkiss. SOLA-VOF: A Solution Algorithm for Transient Fluid Flow with Multiple Free Boundaries. UOC/LA-8355.

R.Purvis and F.Smith. Large Droplet Impact on Water Layers. AIAA-2004-0414.

R. Scardovelli and Stéphane Zaleski. Direct Numerical Simulation of Free-Surface and Interfacial Flow. Annual Review of Fluid Mechanics (1999), vol 31, pp. 567-603.

S. T. Thoroddsen. The Ejecta Sheet Generated by the Impact of a Drop. Journal of Fluid Mechanics (2002), vol. 451, pp. 371-381.

M.D. Torrey, L.D. Cloutman, R.C. Mjolsness and C.W. Hirt. NASA-VOF2D: A Computer Program for Incompressible Flows with Free Surface. LA-10612-MS. UC-32. December 1985.

Trinh et al. Measurement of the density, sound velocity, surface tension and viscosity of freely suspended super cooled liquids. Twelfth Symposium on Thermophysical Properties (1994), June 19-24.

D.A. Weiss and A.L. Yarin. Single Drop Impact onto Liquid Films: Neck Distorsion, Jetting, Tiny Bubble Entrainment and Crown Formation. Journal of Fluid Mechanics (1999), vol. 385, pp. 229-254. 